\section{Manifestaciones cutáneas en donantes de sangre portadores de HTLV-1 en comparación con donantes de sangre no portadores de HTLV-1}

\author{
ISIDORA GARCÍA-HUIDOBRO ${ }^{1}$, CONSUELO CÁRDENAS ${ }^{1}$, \\ MONTSERRAT MOLGÓ', SERGIO GONZÁLEZ ${ }^{2}$
}

\section{Cutaneous manifestations detected in human $\mathrm{T}$ cell lymphotropic virus type 1 infected Chilean blood donors}

Background: Most human T cell lymphotropic virus type 1 (HTLV-1) infected individuals are asymptomatic, but they commonly present cutaneous lesions that could be considered warning signs of the disease. Aim: To identify the main cutaneous manifestations present in HTLV-1 infected blood donors and compare them with healthy donors. Materials and Methods: Two blood donor groups from the blood bank of an emergency hospital were matched according to gender and age. One group was formed by HTLV-1 (+) (cases) and the other by HTLV-1 (-) donors (controls). A blind examiner to the serologic condition, evaluated their cutaneous manifestations. Results: Twenty five cases and 25 controls aged 18 to 60 years (24 females) were evaluated. One or more cutaneous manifestations were found in 24 (96\%) cases and in 15 (60\%) controls $(p<0.01)$. Inflammatory cutaneous diseases were found in $19(76 \%)$ cases and in $9(36 \%)$ controls $(p<0.01)$. Dermatophytosis was found in $18(72 \%)$ cases and in 12 (48\%) controls (NS). Conclusions: HTLV-1 infected Chilean subjects have a higher frequency of dermatoses than their healthy counterparts.

(Rev Med Chile 2014; 142: 859-866)

Key words: Human T-lymphotropic virus 1; Leukemia-Lymphoma, Adult T-Cell; Paraparesis, Spastic.

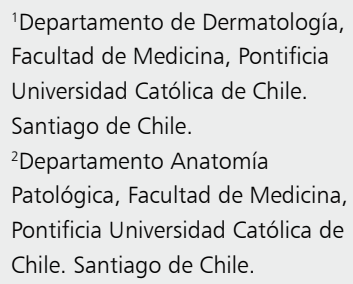

Financiamiento: Departamento de Dermatología, Facultad de Medicina, Pontificia Universidad Católica de Chile.

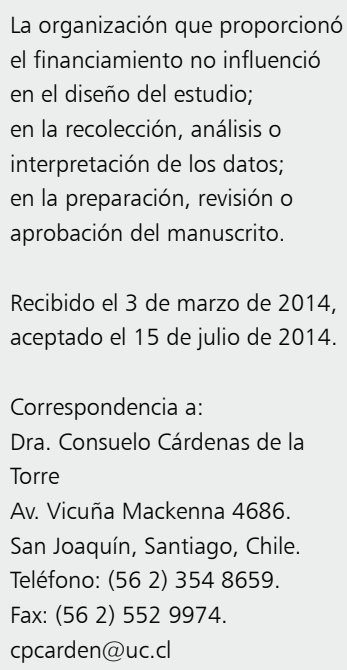

$\mathrm{E}$ 1 virus HTLV-1 (human T cell lymphotropic virus type 1) pertenece al género Deltaretrovirus, familia Retroviridae, subfamilia Oncoviridae. En 1980, el HTLV-1 fue el primer retrovirus patogénico conocido y se aisló desde linfocitos periféricos de un paciente con linfoma T cutáneo ${ }^{1}$.

Se calculan entre 15 y 20 millones de infectados mundialmente ${ }^{2}$. La infección se puede adquirir por vía sexual, vertical o sanguínea ${ }^{3}$. Se han encontrado altas prevalencias (5-10\%) en zonas de Japón, África y el Caribe y muy bajas en Europa y Norteamérica $(<0,01 \%)$. En Sudamérica, se han reportado áreas de Brasil, Colombia y Perú con altas tasas $(>2 \%)^{1}$. En Chile, en un estudio realizado en 954 donantes en el año 1991, se encontró una sero-prevalencia de $0,73 \%{ }^{4}$. Desde enero de 2009 es obligatorio el tamizaje de este virus en los bancos de sangre y todos los casos positivos se confirman en el Instituto de Salud Pública (ISP). Según datos entregados por el ISP, en el período comprendido entre enero de 2009 y julio de 2010, se confirmó la infección por HTLV-1 en un total de 737 donantes de sangre, provenientes de todo el país. El mayor porcentaje de infectados correspondió a mujeres y hombres entre 45 y 55 
años de edad. La sero-prevalencia en la población estudiada fue de $0,16 \%$. Estudios en población indígena chilena muestran prevalencia mucho más altas, que van desde 0,74-1\% en Mapuches, hasta $6,5 \%$ en Aimaras ${ }^{5-7}$.

Aunque la mayoría de los infectados permanece asintomático a lo largo de su vida, el rol etiológico del HTLV-1 se ha establecido en la leucemia/ linfoma de células T del adulto (LLTA) ${ }^{8-12}$, la paraparesia espástica tropical (PPE) $)^{13-17}$, la dermatitis infectiva (DI) ${ }^{18}$ y la uveítis ${ }^{19}$. Se calcula que hasta $5 \%$ de los infectados desarrolla LLTA o PPE, pero la prevalencia e incidencia varían según el área geográfica ${ }^{2}$. El virus también se ha asociado con enfermedades inflamatorias (artropatías, Sjögren, polimiositis, tiroiditis, neumopatías, etc) e infecciosas (estrongiloidiasis, sarna noruega, tuberculosis, dermatofitosis, etc), pero no existen pruebas epidemiológicas suficientes para confirmar estas asociaciones ${ }^{3}$.

Las lesiones cutáneas son comunes en los individuos infectados y pueden representar un signo de alerta para el diagnóstico de la infección.

El compromiso cutáneo en la LLTA está presente en más de $2 / 3$ de los casos, puede ser la primera manifestación clínica en $1 / 3$ y en la mayoría es generalizado. Las lesiones que se pueden encontrar son muy variables, desde máculo-pápulas, parches, placas, nódulos, tumores y eritrodermia ${ }^{20-22}$.

En el caso de la PPE, los pacientes afectados presentan más xerosis, candidiasis cutánea y eritema palmar que la población seronegativa ${ }^{23}$.

La DI es una dermatitis característicamente exudativa, recurrente, que se presenta generalmente en niños, pero también puede observarse en adultos. En general es la primera manifestación de la infección por HTLV-1 en niños y es un marcador de riesgo para el desarrollo posterior de LLTA o PPE. La tasa de incidencia estimada de DI en niños jamaicanos portadores de HTLV-1 es de 552 por 100.000 personas-años. La incidencia de DI es variable según área geográfica, habiendo muchos menos casos reportados en Japón, a pesar de la alta prevalencia del virus ${ }^{24}$.

Se ha descrito asociación entre el HTLV-1 y otras patologías dermatológicas como la dermatitis seborreica, ictiosis adquirida, vitíligo, dermatofitosis, sarna noruega, infecciones bacterianas y virales $^{20,21,25-30}$. Sin embargo, no se conoce con certeza la prevalencia de lesiones dermatológicas en los portadores de HTLV-1. Sólo existe un estudio realizado en Belo Horizonte, Brasil, que reportó que $49 \%$ de los donantes de sangre seropositivos tienen lesiones en la piel en comparación con $12 \%$ de los donantes seronegativos (OR 7,08 $)^{27}$. Las enfermedades dermatológicas más frecuentes en los portadores fueron las dermatofitosis, la ictiosis adquirida y la dermatitis seborreica. Considerando que la prevalencia de la LLTA, PPE y DI son variables según la distribución geográfica, es esperable que las manifestaciones cutáneas en los portadores de HTLV-1 puedan ser distintas también. En Chile no existen estudios al respecto.

Creemos que los portadores de HTLV-1 en Chile también deben presentar variadas lesiones dermatológicas y en mayor frecuencia que los pacientes sero-negativos. En nuestro estudio queremos identificar las principales manifestación cutáneas que presentan los donantes de sangre portadores de HTLV-1; queremos conocer las frecuencias de enfermedades cutáneas infecciosas, inflamatorias y de infiltración neoplásica en ellos y compararlas en relación a donantes sanos.

\section{Materiales y Métodos}

Estudio analítico descriptivo de tipo transversal.

Se estudiaron 2 grupos de donantes del Banco de Sangre del Hospital de Urgencia de Asistencia Pública (HUAP): un grupo denominado "casos", conformado por donantes que en el tamizaje obligatorio hayan resultado positivo a HTLV-1, y que hayan sido confirmados por el ISP, desde enero de 2009 hasta julio de 2012; y el otro grupo denominado "controles", formado por donantes con tamizaje negativo a HTLV-1, desde enero de 2009 a la fecha de realizado el estudio.

No se realizó estimación del tamaño muestral ya que el número de casos era limitado y preestablecido. El número de portadores de HTLV-1 en el Banco de Sangre del HUAP era de 50 personas al momento del estudio. Se incluyeron a todos los que cumplían los criterios de inclusión y accedieron a participar en el estudio. Se excluyeron a los donantes con tamizaje y confirmación positiva de virus de inmunodeficiencia humana, virus hepatitis B y/o virus hepatitis C.

Los grupos fueron pareados en relación 1:1 según: sexo y grupo etáreo (18-30, 31-45 y 46-60 años). 
Se contactaron a los participantes vía telefónica, se les dio a conocer la investigación y se les invitó a participar. Se citaron en el Centro de Especialidades Médicas de la Pontificia Universidad Católica de Chile (PUC), donde se explicó el consentimiento informado, que debieron entender y firmar para ingresar al estudio.

Se registraron los antecedentes demográficos.

Un evaluador, ciego al estado serológico de los participantes, registró antecedentes médicos y antecedentes de enfermedades cutáneas en los últimos 3 meses y realizó un examen físico dermatológico completo.

Se buscó dirigidamente:

- Enfermedades infecciosas (hongos: dermatofitosis, pitiriasis versicolor, candidiasis mucocutánea; parasitarias: escabiosis, pediculosis; bacterianas: piodermias superficiales y profundas; virales: moluscos, verrugas, herpes simplex, herpes zoster).

- Enfermedades inflamatorias (dermatitis seborreica; dermatitis atópica; eccemas: dermatitis de contacto, eccema numular, liquen simple crónico; vitiligo; dermatitis infectiva; xerosis/ ictiosis; aftas orales).

- Infiltración cutánea por LLTA y linfomas cutáneos.

El diagnóstico se basó en criterios clínicos. En algunos casos de cuadro clínico compatible con dermatofitosis, se realizaron micológico directo y cultivo de hongos. En caso de cuadro clínico sospechoso de linfoma cutáneo o de infiltración cutánea por LLTA, se realizó estudio histopatológico de piel para confirmar el diagnóstico.

Todos los procedimientos realizados respetaron normas éticas concordantes con la Declaración de Helsinki y fueron revisados y aprobados por el Comité Ético Científico de la PUC.

\section{Estadística}

Se realizó el análisis descriptivo de las variables en estudio y se utilizó el Test de Probabilidad Exacta de Fisher para evaluar la asociación entre variables nominales. Se consideró estadísticamente significativo valores $\mathrm{p} \leq 0,05$. Además se calculó Odds ratios (ORs) e intervalos de confianza de 95\% para determinar y cuantificar las asociaciones. Los datos fueron analizados usando el programa en línea VassarStats.

\section{Resultados}

De los 50 casos se pudo contactar y aceptaron participar 25 . Se analizaron 25 casos y 25 controles, pareados por sexo y grupo etáreo. La descripción de la muestra se resume en la Tabla 1.

En total se estudiaron 24 hombres y 26 mujeres con un promedio de edad de 45 años. La mayoría (60\%) pertenecía al grupo etáreo 46-60 años.

Siete participantes pertenecían a alguna etnia originaria o eran de nacionalidad extranjera: 3 peruanos, 2 sirios y 2 mapuches. Todos ellos eran casos, excepto 1 participante mapuche que era

Tabla 1. Descripción de la muestra

\begin{tabular}{|lccc|}
\hline & Total & Casos & Controles \\
\hline Sexo & & & 12 \\
$\quad$ Hombres & 24 & 12 & 13 \\
$\quad$ Mujeres & 26 & & 3 \\
Grupo etario & & 3 & 7 \\
$\quad 18-30$ años & 6 & 7 & 15 \\
31-45 años & 14 & 15 & 1 \\
46-60 años & 30 & 6 & 10 \\
Etnia originaria o nacionalidad extranjera & 7 & 10 & 6 \\
Antecedentes médicos variados & 20 & 6 & 2 \\
Alergia de contacto a metales & 12 & 7 & 3 \\
Transfusiones sanguíneas & 9 & 14 & \\
\hline Enfermedades cutáneas en los últimos 3 meses & 17 & & \\
\hline
\end{tabular}


Manifestaciones cutáneas en donantes de sangre portadores de HTLV-1 - I. García-Huidobro et al

Tabla 2. Comparación entre casos y controles según tipo manifestación cutánea

\begin{tabular}{|c|c|c|c|c|c|}
\hline Manifestación & Casos & Controles & Valor p & & OR \\
\hline \multicolumn{6}{|l|}{ Infecciosas } \\
\hline Dermatofitosis & $18(72 \%)$ & 12 (48\%) & 0,074 & 2,79 & $(0,86-9)$ \\
\hline Candidiasis & $2(8 \%)$ & $0 \quad(0 \%)$ & 0,245 & & - \\
\hline Pitiriasis versicolor & $1 \quad(4 \%)$ & 0 & 0,5 & & - \\
\hline Corynebacterium & $1 \quad(4 \%)$ & 0 & 0,5 & & c \\
\hline Verrugas & $2 \quad(8 \%)$ & $1 \quad(4 \%)$ & 0,5 & 2,087 & $7(0,18-24,6)$ \\
\hline Total personas & 18 (72\%) & 12 (48\%) & 0,074 & 2,79 & $(0,86-9)$ \\
\hline Total manifestaciones & 24 & 13 & & & \\
\hline \multicolumn{6}{|l|}{ Inflamatorias } \\
\hline Xerosis/Ictiosis & $10 \quad(40 \%)$ & $4 \quad(16 \%)$ & 0,057 & 3,5 & $(0,92-13,3)$ \\
\hline Dermatitis seborreica & 7 (28\%) & $4 \quad(16 \%)$ & 0,248 & 2,0 & $(0,5-8,1)$ \\
\hline Eccema & 7 (28\%) & $2 \quad(8 \%)$ & 0,069 & 4,47 & $(0,83-24,2)$ \\
\hline Vitiligo & $2 \quad(8 \%)$ & 0 & 0,245 & & - \\
\hline Psoriasis & $1 \quad(4 \%)$ & $1 \quad(4 \%)$ & 0,755 & 1 & $(0,06-16,9)$ \\
\hline Total personas & 19 (76\%) & $9 \quad(36 \%)$ & 0,0048 & 5,6 & $(1,65-19,2)$ \\
\hline Total manifestaciones & 27 & 11 & & & \\
\hline
\end{tabular}

control. Nueve participantes recibieron transfusiones sanguíneas antes del año 2009 (7 casos y 2 controles).

Catorce casos $(58 \%)$ y 3 controles $(12 \%)$ referían haber tenido alguna enfermedad cutánea en los últimos 3 meses, diferencia con significancia estadística $(\mathrm{p}=0,0011)$.

Se encontró una o más de las manifestaciones cutáneas buscadas en $24(96 \%)$ casos y en 15 $(60 \%)$ controles, con una diferencia estadísticamente significativa $(\mathrm{p}=0,0023)$.

Al comparar la presencia de enfermedades infecciosas, se encontraron $18(72 \%)$ casos y $12(48 \%)$ controles, sin diferencia significativa $(p=0,074)$. Todos los participantes que presentaron infección, presentaban dermatofitosis. El resto de infecciones cutáneas encontradas se describen en la Tabla 2. El diagnóstico de dermatofitosis se hizo de manera clínica. A 19 personas (12 casos y 7 controles) se les realizó micológico directo y cultivo de hongos. Al resto no se les realizó por ser únicamente interortejos o porque no acudieron para la realización de éste o por estar en tratamiento. En 16 personas el laboratorio fue positivo (10 casos y 6 controles). Los cultivos que resultaron positivos mostraron desarrollo de Trichophyton rubrum en 6 participantes ( 3 casos y 3 controles), de Trichophyton mentagrophytes en otros 3 (2 casos y 1 control) y de Candida sp en uno ( 1 control en una muestra de planta de pie).
Se encontraron $19(76 \%)$ casos y $9(36 \%)$ controles con enfermedades inflamatorias, con diferencia estadísticamente significativa $(\mathrm{p}=0,0048)$. El análisis de subgrupos se muestra en la Tabla 2. No hubo diferencia significativa en éstos.

Hubo una mujer de 48 años, portadora de HTLV-1, con historia de 6 meses de lesiones generalizadas eritemato-descamativas pruriginosas, refractarias al tratamiento con corticoides orales y tópicos (Figuras 1 y 2). En ella se sospechó micosis fungoide, pero el estudio anatomopatológico de 5 muestras fue compatible con dermatitis perivascular superficial linfocitaria leve. Este caso presentaba además dermatofitosis y xerosis/ictiosis. En el examen físico pesquisamos alteración en la marcha y se derivó a neurología, donde se diagnosticó Paraparesia espástica secundaria a HTLV-1.

No se sospechó infiltración cutánea por LLTA en ningún participante.

\section{Discusión}

Este es el primer estudio chileno que describe las manifestaciones cutáneas en donantes de sangre portadores de HTLV-1. Además se comparó con donantes seronegativos. Existe un solo estudio de Gonçalves et al que realiza una comparación similar en Belo Horizonte, Brasil ${ }^{27}$ y un estudio que busca manifestaciones cutáneas en pacientes 


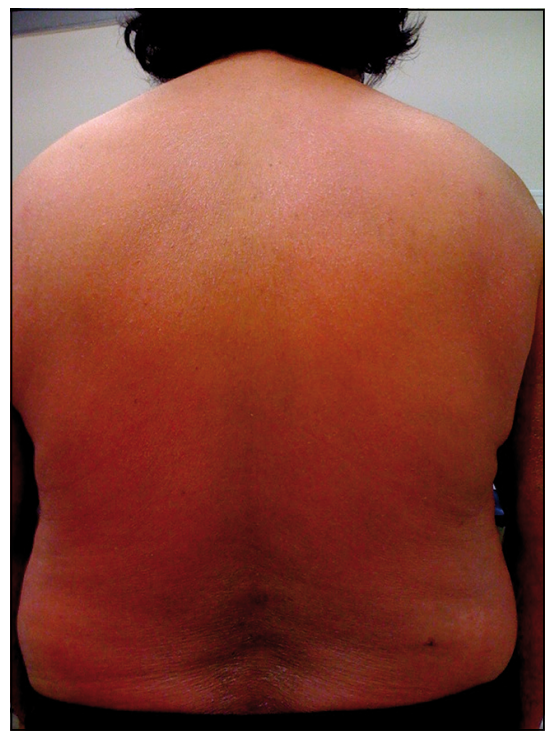

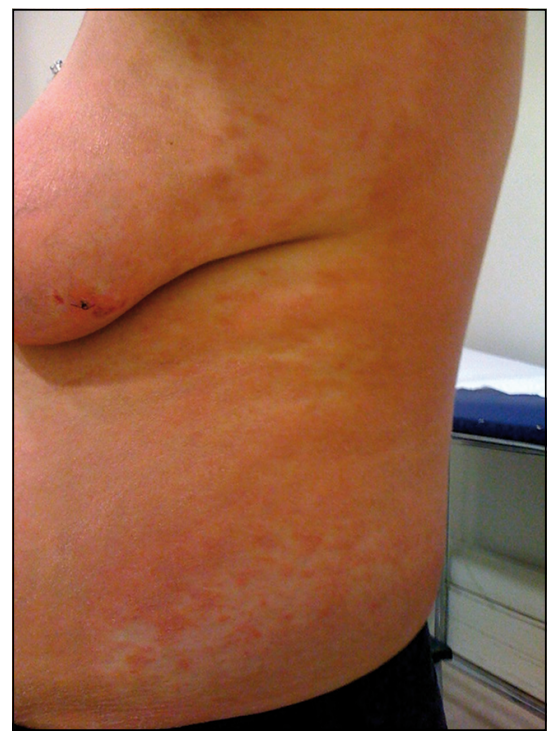

Figura 1 y 2. Mujer portadora de HTLV-1 con parches eritemato-descamativos generalizados.
PPE asociada a HTLV-1 y los compara con controles sanos ${ }^{23}$.

$\mathrm{Al}$ igual que en el estudio de Gonçalves et al, los casos seropositivos presentaron más alteraciones dermatológicas que el grupo control ( $96 \%$ vs $60 \%$ ), siendo la diferencia estadísticamente significativa en ambos estudios. Además en nuestro estudio, los casos referían más enfermedad cutáneas en los últimos 3 meses que los controles ( $58 \%$ vs $12 \%)$, diferencia que también tuvo significancia estadística.

En nuestro estudio el grupo de manifestaciones cutáneas más frecuentes en los portadores de HTLV-1 fueron las inflamatorias, con diferencia significativa en relación a los controles $(76 \%$ vs $36 \%$ ). La diferencia no fue significativa al analizar los subgrupos. Sin embargo, se observó una clara tendencia a presentar mayor xerosis/ictiosis y eccemas.

En nuestro estudio la xerosis/ictiosis fue la manifestación inflamatoria más frecuente y se encontró en $40 \%$ de los casos y $16 \%$ de los controles, en comparación con el estudio de Gonçalves et al en que se encontró en $8,8 \%$ y $0 \%$ respectivamente, encontrándose diferencia estadísticamente significativa en ese estudio. Se han planteado 2 teorías que podrían explicar la mayor prevalencia de xerosis/ictiosis en esta población: 1) Daño de la glándula ecrina por un infiltrado inflamatorio linfocitario y 2) Alteración de la regulación au- tonómica de las glándulas, por daño neuronal. Esta última teoría se aplicaría en los pacientes con $\mathrm{PPE}^{23,27,31}$.

Cabe destacar, que los portadores de HTLV-1 presentaban más de una manifestación inflamatoria por persona de forma más frecuente que los controles, quienes en general presentaban sólo una manifestación a la vez. Esta mayor predisposición a la inflamación, ha sido explicada en algunos estudios por un aumento en los niveles séricos de citoquinas proinflamatorias, como el TNF alfa e INF gamma, encontrada en portadores de HTLV1 y una desregulación del sistema inmune $e^{26,32-34}$.

Las manifestaciones infecciosas fueron casi tan frecuentes como las inflamatorias en los portadores de HTLV-1. La diferencia en comparación con el grupo control no fue estadisticamente significativa, sin embargo, mostró una tendencia ( $72 \%$ vs $48 \%$, OR 2,79 ). Todos los participantes que presentaron alguna infección, presentaban dermatofitosis, en algunos casos con coinfecciones. En el estudio de Gonçalves et al, la dermatofitosis se encontró en 34,4\% de los seropositivos y en $14,8 \%$ de los controles, con una diferencia estadísticamente significativa. Como se observa, nuestros controles tienen mucho mayor prevalencia de dermatofitosis que los controles del estudio brasilero ( $48 \%$ vs $14,8 \%$ ). Un estudio chileno, en población de riesgo (reclutas militares en Valdivia) mostró una prevalencia de $29,3 \%$ de 
dermatofitosis, lo cual también es menor a la prevalencia de nuestro grupo control ${ }^{35}$. Esto se puede explicar por un sesgo de selección de los controles, debido a que al menos $70 \%$ de los controles citados al estudio no acudían a su hora. Nos impresiona que una parte importante de los controles que participaron efectivamente del estudio, lo hicieron motivados por presentar alguna patología cutánea que no habían resuelto, siendo la dermatofitosis la patología más frecuente de este grupo. Las especies de hongos que se desarrollaron con mayor frecuencia en ambos grupos fueron Trichophyton rubrum y Trichophyton mentagrophytes, lo que concuerda con lo publicado en distintos trabajos nacionales ${ }^{36,37}$.

Al igual que en las manifestaciones inflamatorias, los portadores de HTLV-1 presentaban más de una manifestación infecciosa por persona de forma más frecuente que los controles, quienes en general presentaban sólo una a la vez.

Llama la atención el caso de la mujer de 48 años, portadora de HTLV-1, que presentaba una dermatitis generalizada refractaria al tratamiento, xerosis/ictiosis y dermatofitosis y que se le diagnosticó además una paraparesia espástica. Nosotros repetimos el tratamiento con corticoides tópicos, orales, antihistamínicos, medidas generales y suspensión de contactantes, pero no obtuvimos respuesta satisfactoria. La paciente tiene hemograma y perfil bioquímico normales. Se han descrito en la literatura dermatosis inflamatorias persistentes en portadores de HTLV-1, que histológicamente corresponden a infiltrado linfocitario sin células atípicas. Esto podría ser explicado parcialmente por los elevados niveles de citoquinas pro-inflamatorias que pueden tener los sero-positivos. Algunos autores postulan que estos casos podrían progresar a linfoma, por lo que deberían ser controlados periódicamente ${ }^{21}$.

Considerando que la prevalencia de la LLTA, PPE y DI son variables según la distribución geográfica, podía esperarse que las manifestaciones cutáneas en los portadores de HTLV-1 fueran distintas también. La importancia de este estudio es que permite tener un primer acercamiento al conocimiento de las principales dermatosis en chilenos portadores de HTLV-1 y confirma que son más frecuentes que en los donantes sanos.

La principal limitación de este estudio se encuentra el tamaño muestral pequeño que pudiera influir en los resultados, al disminuir la probabi- lidad de encontrar diferencias entre los grupos; y el sesgo de selección de los controles.

Para profundizar en el conocimiento de este tema, habría que realizar un estudio multicéntrico, que involucrara a donantes de distintos bancos de sangre del país representativos de la población general y probablemente dar un incentivo a los controles para evitar el sesgo de selección mencionado anteriormente.

Agradecimientos: Agradecemos a la Dirección del Hospital de Urgencia de Asistencia Pública (HUAP) y al personal del Banco de Sangre del HUAP que permitieron que este estudio pudiera llevarse acabo.

\section{Referencias}

1. Poiesz BJ, Ruscetti FW, Gazdar AF, Bunn PA, Minna JD, Gallo RC. Detection and isolation of type $\mathrm{C}$ retrovirus particles from fresh and cultured lymphocytes of a patient with cutaneous T-cell lymphoma. Proc Natl Acad Sci USA 1980; 77 (12): 7415-9.

2. Proietti FA, Carneiro-Proietti AB, Catalan-Soares BC, Murphy EL. Global epidemiology of HTLV-I infection and associated diseases. Oncogene 2005; 24 (39): 605868.

3. Verdonck K, González E, Van Dooren S, Vandamme AM, Vanham G, Gotuzzo E. Human T-lymphotropic virus 1: recent knowledge about an ancient infection. Lancet Infect Dis 2007; 7 (4): 266-81.

4. Vázquez P, Sánchez G, Volante C, Vera L, Ramírez E, Soto G, et al. Human T Lymphotropic Virus Type I: New risk for Chilean population (letter). Blood 1991; 78 (3): 850-1.

5. Ministerio de Salud. Circular B21-32 (2008) Instruye a los centros de bancos de sangre de la red asistenciales de salud a pesquisar el virus HTLV-1.

6. http://www.ispch.cl/notacientifica/14238/virus-linfotropico-de-celulas-t-humano-tipo-i-y-ii-htlv-iii

7. Cartier L, Tajima K, Araya F, Castillo JL, Zaninovic V, Hayami M, et al. [Preliminary study of HTLV-I seroprevalence in Chilean Indian populations]. Rev Med Chile 1993; 121 (3): 241-6.

8. Hinuma Y, Nagata K, Hanaoka M, Nakai M, Matsumoto T, Kinoshita KI, et al. Adult T-cell leukemia: antigen in an ATL cell line and detection of antibodies to the antigen in human sera. Proc Natl Acad Sci USA 1981; 78 (10): 6476-80.

9. Miyoshi I, Miyamoto K, Sumida M, Nishihara R, Lai 
M, Yoshimoto, et al. Chromosome $14 \mathrm{q}+$ in adult T-cell leukemia. Cancer Genet Cytogenet 1981; 3 (3): 251-9.

10. Yoshida M, Seiki M, Yamaguchi K, Takatsuki K. Monoclonal integration of human T-cell leukemia provirus in all primary tumors of adult T-cell leukemia suggests causative role of human T-cell leukemia virus in the disease. Proc Natl Acad Sci USA 1984; 81 (8): 2534-7.

11. Takatsuki K, Yamaguchi K, Kawano F, Hattori T, Nishimura $\mathrm{H}$, Tsuda $\mathrm{H}$, et al. Clinical diversity in adult T-cell leukemia-lymphoma. Cancer Res 1985; 45 (9 Suppl): 4644s-5s.

12. Takatsuki K, Yamaguchi K, Kawano F, Nishimura H, Seiki M, Yoshida M. Clinical aspects of adult T-cell leukemia/lymphoma. Curr Top Microbiol Immunol 1985; 115: 89-97.

13. Cruikshank EK. A neuropathic syndrome of uncertain origin; review of 100 cases. West Indian Med J 1956; 5 (3): 147-58.

14. Gessain A, Barin F, Vernant JC, Gout O, Maurs L, Calender A, et al. Antibodies to human T-lymphotropic virus type-I in patients with tropical spastic paraparesis. Lancet 1985; 2 (8452): 407-10.

15. Rodgers-Johnson P, Gajdusek DC, Morgan OS, Zaninovic V, Sarin PS, Graham DS. HTLV-I and HTLV-III antibodies and tropical spastic paraparesis. La ncet. 1985; 2 (8466): 1247-8.

16. Osame M, Usuku K, Izumo S, Ijichi N, Amitani H, Igata A, et al. HTLV-I associated myelopathy, a new clinical entity. Lancet 1986; 1 (8488): 1031-2.

17. Rodgers PE. The clinical features and aetiology of the neuropathic syndrome in jamaica. West Indian Med J 1965; 14: 36-47.

18. La Grenade L, Hanchard B, Fletcher V, Cranston B, Blattner W. Infective dermatitis of Jamaican children: a marker for HTLV-I infection. Lancet 1990; 336 (8727): 1345-7.

19. Mochizuki M, Watanabe T, Yamaguchi K, Tajima K, Yoshimura K, Nakashima S, et al. Uveitis associated with human $\mathrm{T}$ lymphotropic virus type I: seroepidemiologic, clinical, and virologic studies. J Infect Dis 1992; 166 (4): 943-4.

20. Bittencourt AL, de Oliveira M de F. Cutaneous manifestations associated with HTLV-1 infection. Int J Dermatol 2010; 49 (10): 1099-110.

21. Bittencourt AL, da Graças Vieira M, Brites CR, Farre L, Barbosa HS. Adult T-cell leukemia/lymphoma in Bahia, Brazil: analysis of prognostic factors in a group of 70 patients. Am J Clin Pathol 2007; 128 (5): 875-82.

22. Bittencourt AL, Barbosa HS, Vieira MD, Farré L. Adult T-cell leukemia/lymphoma (ATL) presenting in the skin: clinical, histological and immunohistochemical features of 52 cases. Acta Oncol 2009; 48 (4): 598-604.

23. Lenzi ME, Cuzzi-Maya T, Oliveira AL, Andrada-Serpa MJ, Araújo AQ. Dermatological findings of human T lymphotropic virus type 1 (HTLV-1) associated myelopathy/tropical spastic paraparesis. Clin Infect Dis 2003; 36 (4): 507-13.

24. Maloney EM, Hisada M, Palmer P, Brooks K, Pate E, Wiktor SZ, et al. Human T cell lymphotropic virus type I-associated infective dermatitis in Jamaica: a case report of clinical and biologic correlates. Pediatr Infect Dis J 2000; 19 (6): 560-5.

25. Molgó M, Giesen L, Giácaman M, González S. Manifestaciones cutáneas de la infección por HTLV-1. Piel 2012; 27 (6): 327-32.

26. Amano M, Setoyama M, Grant A, Kerdel FA. Human T-lymphotropic virus 1 (HTLV-1) infection--dermatological implications. Int J Dermatol 2011; 50 (8): 915-20.

27. Gonçalves DU, Guedes AC, Proietti AB, Martins ML, Proietti FA, Lambertucci JR. Interdisciplinary HTLV-1/2 Research Group. Dermatologic lesions in asymptomatic blood donors seropositive for human $\mathrm{T}$ cell lymphotropic virus type-1. Am J Trop Med Hyg 2003; 68 (5): 562-65.

28. Pezeshkpoor F, Yazdanpanah MJ, Shirdel A. Specific cutaneous manifestations in adult T-cell leukemia/ lymphoma. Int J Dermatol 2008; 47 (4): 359-62.

29. Brites C, Weyll M, Pedroso C, Badaro R. Severe and Norwegian scabies are strongly associated with retroviral (HIV-/HTLV-1) infection in Bahia, Brazil. AIDS 2002; 16 (9): 1292-93.

30. Blas M, Bravo F, Castillo WJ, Ballona R, Navarro P, Catacora J, et al. Norwegian scabies in Peru: the impact of human T cell lymphotropic virus type I infection. Am J Trop Med Hyg 2005; 72 (6): 855-7.

31. Setoyama M, Mizoguchi S, Eizuru Y. Human T-cell lymphotropic virus type I infects eccrine sweat gland epithelia. Int J Cancer 1999; 80 (5): 652-5.

32. Carvalho EM, Bacellar O, Porto AF, Braga S, GalvãoCastro B, Neva F. Cytokine profile and immunomodulation in asymptomatic human T-lymphotropic virus type 1-infected blood donors. J Acquir Immune Defic Syndr 2001; 27 (1): 1-6.

33. Santos SB, Porto AF, Muniz AL, de Jesus AR, Magalhães E, Melo A, et al. Exacerbated inflammatory cellular immune response characteristics of HAM/TSP is observed in a large proportion of HTLV-I asymptomatic carriers. BMC Infect Dis 2004; 4: 7.

34. Nascimento MC, Primo J, Bittencourt A, Siqueira I, de Fátima Oliveira M, Meyer R, et al. Infective dermatitis has similar immunological features to human $\mathrm{T}$ lym- 
photropic virus-type 1-associated myelopathy/tropical spastic paraparesis. Clin Exp Immunol 2009; 156 (3): 455-62.

35. Zaror L, Lorca S, Martínez C. Dermatophytes in soldiers in risk areas at Valdivia regiments. Bol Micol 1991; 6 (1/2): 27-31.

36. Díaz MC, Fich F, Salamanca L, Hering M. Variaciones en la etiología de las micosis superficiales en dos servicios hospitalarios de la Región Metropolitana. Rev Med Chile 1987; 115: 319-22.

37. Fich F, Díaz MC, Moreno MI, Salamanca L. Dermatomicosis superficiales: Estudio en pacientes del Servicio Metropolitano Central Santiago. Rev Med Chile 1981; 109: 735-39. 